\title{
Impacto de los hábitos sobre la mineralización ósea durante la etapa de la juventud
}

\author{
Paola Orozco Santos ${ }^{1}$; John Jairo Duque ${ }^{2}$; Juan Carlos Lucas ${ }^{3}$
}

${ }^{1}$ (Enfermera, MSc Ciencias Biomédicas, Corporación Universitaria Empresarial Alexander von Humboldt)

${ }^{2}$ (MD Endocrinólogo, Corporación Universitaria Empresarial Alexander von Humboldt)

${ }^{3}$ (Ingeniero Industrial, candidato a Doctor en Ingeniería de Alimentos, Corporación Universitaria Empresarial Alexander von Humboldt)

Fecha de recepción: 25/08/2015

Fecha de aceptación: 30/09/2015

\section{Resumen}

$\mathrm{L}$ os mayores picos de mineralización se producen en los primeros 2 años de vida y la pubertad, en los que la masa ósea aumenta $40 \%$ y $60 \%$; a los 20 años se estabiliza y, luego, se inicia una pérdida progresiva. El volumen de masa ósea es variable según la edad, el sexo y la raza, es afectado por factores ambientales, genéticos, hormonales y nutricionales. El objetivo del estudio fue conocer el impacto de los hábitos relacionados con el proceso de mineralización durante la etapa de la juventud. Los participantes completaron una ficha clínica. La densidad mineral ósea fue valorada por medio de densitometría, se midieron los niveles de calcio en orina para identificar posibles pérdidas, se calculó el índice de masa corporal (IMC) a partir del peso y la talla. El $5 \%$ de la población participante presentó un Z-score inferior a -2,0 y los factores relacionados fueron consumo de cafeína, alcohol y poca actividad física.

Palabras Clave: mineralización ósea, calcio en huesos, osteoporosis, densidad mineral ósea.

\section{Abstract}

The highest peaks of mineralization is in the first two years of life and puberty, bone mass increases $40 \%$ to $60 \%$, at twenty stabilizes and begins a gradual loss. The volume of bone mass varies according to age, sex and race, which affect environmental, genetic, hormonal and nutritional factors. The aim of the study was to determine the impact of the habits related mineralization process during the stage of youth. Participants completed clinical record. BMD was assessed by densitometry; Calcium levels were measured in urine for loss, BMI was calculated from weight and height. 5\% of the participating population present a $Z$-score less than -2,0 and related factors were caffeine, alcohol and little physical activity.

Keywords: bone mineralization, calcium in bones, osteoporosis, bone mineral density.

\section{Introducción}

En condiciones normales, la mineralización del sistema óseo en el humano se inicia durante el tercer trimestre de la gestación y va incrementándose progresivamente durante los primeros 20 años de vida, los mayores picos de mineralización se dan durante los primeros 2 años y en la pubertad, cuando la masa ósea aumenta entre $40 \%$ y $60 \%$. A los 20 años el proceso de mineralización se estabiliza y se inicia una pérdida progresiva de masa ósea en los huesos del fémur y la columna lumbar, la cual se estima alrededor de 0,5 y $1 \%$ anual; en la mujeres antes y después de la menopausia esta perdida es mayor en comparación con los hombres ${ }^{(1)}$. El volumen de masa ósea es variable según la edad, el sexo y la raza, y puede verse afectado fácilmente por factores ambientales, genéticos, hormonales y nutricionales durante la etapa de formación ósea, ocasionando una baja densidad cuando se llega a la maduración del sistema esquelético aumentando con ello el riesgo de aparición de fracturas osteoporóticas durante la vida adulta ${ }^{(2)}$.

La osteoporosis en una enfermedad del sistema óseo generalmente de los adultos, ocasionada por una pérdida progresiva de los depósitos de calcio en los huesos, ocasionando huesos delgados y frágiles, los cuales se fracturan fácilmente y tardan mucho tiempo en repararse ${ }^{(3)}$. Con frecuencia, la osteoporosis se asocia con sarcopenia, una enfermedad que se manifiesta con debilidad muscular, la combinación de sarcopenia y osteoporosis es con frecuencia fatal, ya que aumenta el riesgo de sufrir fracturas de cadera y de columna vertebral, la asociación entre ambas enfermedades se da por factores como el sedentarismo, el poco consumo de proteínas en la dieta y cambios hormonales ${ }^{(4)}$. La osteoporosis también puede ser inducida por consumo de medicamentos como: glucocorticoides, estimulantes de la glándula tiroides y algunos anticoagulantes como la heparina, por enfermedades como: hipertiroidismo, hipotiroidismo, síndrome de Cushing y artritis reumatoide, porque interfieren con el metabolismo del calcio; el consumo de tabaco cafeína y alcohol inducen a pérdida de calcio en orina, y como factor nutricional se asocian a osteoporosis el bajo consumo de alimentos ricos en vitamina $\mathrm{D}$ y calcio ${ }^{(3)}$. La importancia clínica de la osteoporosis reside en que, con el tiempo, las fracturas frecuentes causan dolor, limitación física y cambios en el estilo de vida ${ }^{(5)}$. 
La osteoporosis es un problema de salud pública que afecta a 200 millones de personas en el mundo ${ }^{(6)}$, durante el 2012 en Estados Unidos cerca de 4 millones de mujeres y 1 millón de hombres mayores de 50 años fueron diagnosticados con esta enfermedad ${ }^{(3)}$. En México la prevalencia de osteoporosis es de $16 \%$ y de densidad mineral ósea (DMO) baja de $57 \%$ en mujeres mayores de 50 años ${ }^{(7)}$, de acuerdo con las proyecciones para el año 2050 más del $75 \%$ de las fracturas de cadera ocurrirán en Asia y América Latina, como consecuencia del aumento en la esperanza de vida y el mayor número de personas mayores de 65 años ${ }^{(8)}$.

La determinación de la DMO mediante densitometría por absorción dual de Rx (DEXA) es la prueba gold estándar para el diagnóstico de la osteoporosis. La Organización Mundial de la Salud (OMS) establece los criterios para interpretar los resultados de densitometría ósea, tomando como base el reporte del T-score o el Z-score (Normal: T-score entre +1 y $-1 \mathrm{DE}$ del promedio de población adulta joven, Osteopenia: T-score entre -1 y -2,5 DE del promedio de población adulta joven, Osteoporosis: T-score bajo $-2,5 \mathrm{DE}$ del promedio de población adulta joven, Osteoporosis grave: Z-score DMO bajo -2,5 DE del promedio de población adulta joven y una o más fracturas de tipo osteoporótico) $^{(9)}$.

Aunque existen dificultades para diagnosticar osteoporosis en mujeres premenopáusicas, niños y hombres menores de 50 años, un dato útil para indicar una baja densidad mineral ósea para edad cronológica es un Z-score inferior a -2,0, porque a este nivel se incrementa el riesgo de fracturas, aunque el pico de masa ósea es alcanzado más temprano en las mujeres que en los hombres, reflejando la temprana complementación de la pubertad, en ambas poblaciones, este pico es alcanzado primero en la cadera, después en la columna y finalmente en el cuerpo total ${ }^{(10,11)}$.

En adultos jóvenes que sufren cualquier enfermedad que afecte el metabolismo del calcio, un T-score menor a -2,5 en los huesos de la columna o cadera puede ser considerado para el diagnóstico de la osteoporosis ${ }^{(11)}$.

Estudios previos han demostrado que los estilos de vida son una de las principales causas para el desarrollo de osteoporosis en la vida adulta, pero no es muy conocido o no está muy documentado a partir de qué edad puede empezar esa pérdida de masa ósea, antes de la manifestación clínica de la enfermedad, es por esto que el propósito de este estudio fue conocer si los hábitos afectan el proceso de mineralización ósea durante la etapa de la juventud, con el fin de obtener datos objetivos que orienten actividades de promoción de la salud ósea en personas jóvenes y prevención de la osteoporosis durante la vida adulta.

\section{Metodología}

Se realizó un estudio descriptivo-exploratorio, en jóvenes universitarios entre los 18 y 25 años, de la ciudad de Armenia,
Quindío. El tamaño de la muestra fue calculado de acuerdo con la prevalencia de osteopenia en adultos que es entre $45 \%$ y $49 \%$ según estudios previos (Clark P, et al., 2009) con un margen de error del $10 \%$ y un $95 \%$ de confiabilidad $n=98$. Los criterios de inclusión fueron: estudiantes universitarios de ambos sexos de 18 a 25 años de edad, sin antecedentes personales de enfermedad sistémica y aceptación voluntaria para participar en el estudio. Los criterios de exclusión fueron: mujeres en estado de embarazo o en periodo de lactancia, índice de masa corporal inferior a 16, antecedentes personales o familiares de enfermedades como hipertiroidismo, hipotiroidismo, cáncer, artritis reumatoide, síndrome de Cushing, insuficiencia renal crónica, diabetes mellitus e hipertensión arterial. La valoración nutricional se hizo a través del IMC, tomando como referencia los parámetros establecidos por la OMS. Los participantes completaron una ficha clínica médica-nutricional con el objeto de evaluar los criterios de inclusión y obtener los datos personales y ginecológicos, antecedentes familiares y personales de enfermedades crónicas, estilos de vida como: fumar, consumo de alcohol, cafeína (café y refrescos de cola) y calcio por medio de un recordatorio de 24 horas, se indagó la realización de actividad física y valoración del IMC a través del peso y la talla, la densidad mineral ósea se obtuvo por medio de densitometría dual de Rx con un densitómetro marca HOLOGIC Explorer QDR*. Para el análisis de los datos se utilizó como referencia el Z-score de columna lumbar L1-L4, se realizó una prueba de tamizaje en orina para identificar pérdidas de calcio. Se realizó un análisis estadístico multivariado, utilizando el paquete estadístico Statgraphics Centurion, se tomaron los datos con $\mathrm{p}<0,005$ para establecer el nivel de significancia. Todos los datos obtenidos quedaron consignados en la ficha, la cual fue guardada y custodiada por los investigadores, cada participante fue identificado por medio de un código, desde el 001 hasta el 100. Esto se hizo con el fin de guardar y respetar la confidencialidad del participante.

\section{Resultados}

Se quiso identificar el estado de la salud ósea de la población en términos de masa ósea, para efectos del análisis la población objetivo se dividió en dos grupos, los cuales se denominaron adultos y pediátricos. El grupo de adultos corresponde a 47 personas entre los 20 y 25 años de edad, cuya ubicación se encuentra en la parte superior de la figura 1 representado por barras de color azul claro; el grupo de pediátricos está conformado por 51 personas con un rango de edad entre los 18 y 20 años y se encuentra ubicado en la parte inferior de la figura 1 representado por las barras de color azul oscuro. El valor mínimo del $Z$-score para la población adulta fue de -3 y el valor máximo fue de 2; para la población pediátrica el valor mínimo del $Z$-score fue de -2,6 y el valor máximo de 1,7.

La media del Z-score para la población adulta fue de 0,06 y la desviación estándar de 0,97; para la población pediátrica 
Figura 1. Representación del Z-score en la población objeto de estudio

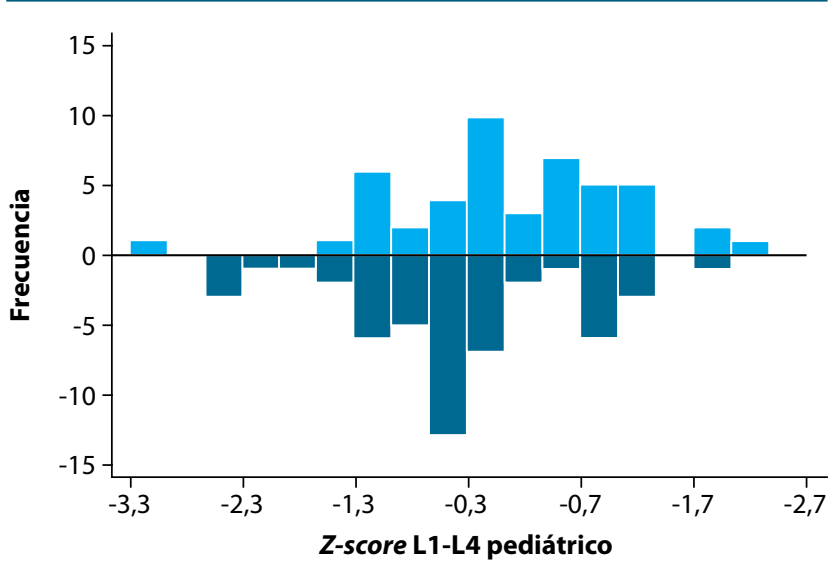

El grupo de adultos corresponde a 47 personas entre los 20 y 25 años de edad, cuya ubicación se encuentra en la parte superior de la figura 1 representado por barras de color azul claro, el grupo de pediátricos está conformado por 51 personas con un rango de edad entre los 18 y 20 años y se encuentra ubicado en la parte inferior de la figura 1 representado por las barras de color azul oscuro.

Figura 2. Tendencias de medida central entre las dos categorías de población adulta y pediátrica

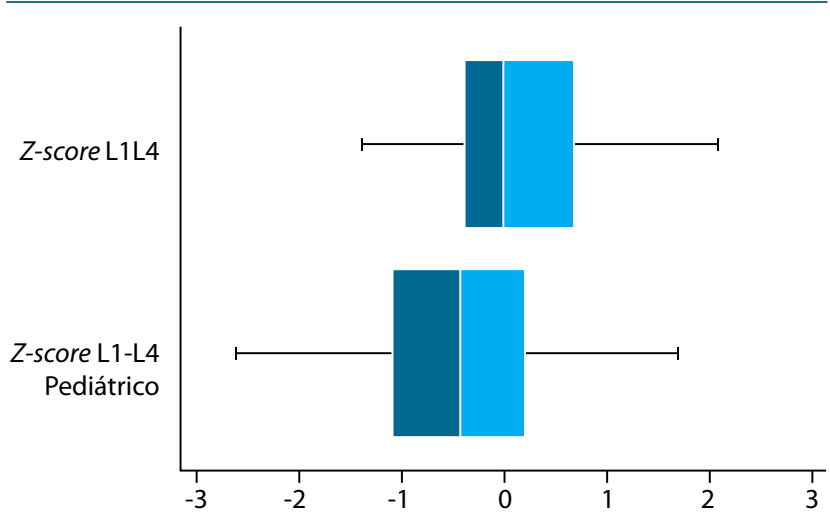

Media muestral del Z-score población adulta entre 20 y 25 años y población pediátrica entre 18 y 20 años. Las cifras del Z-score son mayores en la población adulta.

la media muestral para el Z-score fue de -0,39 y la desviación estándar de 0,99. Con un intervalo de confianza del 95\% y un p-valor inferior a 0,05 se pudo determinar que existe una diferencia estadísticamente significativa entre la media muestral de los dos grupos poblacionales; sin embargo, la diferencia en la desviación estándar no fue estadísticamente significativa (figura 2). En la figura 3 puede observarse que el 5\% de la poblacion analizada presentó un Z-Score inferior a - 2,0, de los cuales el $80 \%$ fueron hombres entre los 18 y 20 años de edad. Una vez identificados los niveles de masa ósea, se analizaron, en toda la población, los niveles de calcio excretados en orina ocasional, como método de tamizaje para identificar posibles pérdidas. El $63 \%$ tuvo niveles de calcio en orina por encima de los valores de referencia, de ellos el $20 \%$ correspondió a mujeres (figura 4). Utilizando una regresión lineal simple, es-
Figura 3. Secuenciación cronológica del Z-score para población adulta y pediátrica
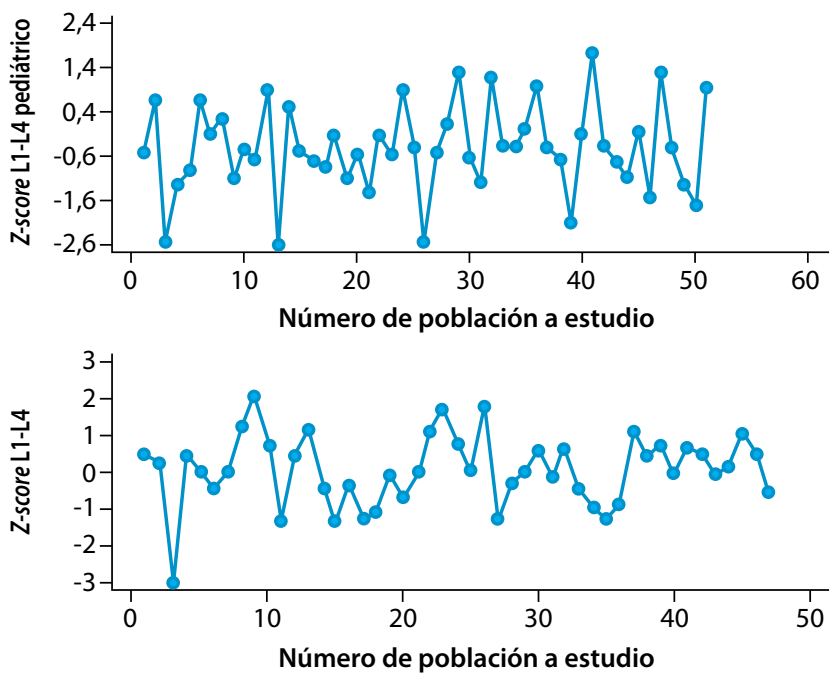

Población con Z-score inferior a -2,0 distribuidos según edad, población adulta entre 20 y 25 años ( 1 sujeto) y población pediátrica entre 18 y 20 años (4 sujetos).

Figura 4. Pacientes que demuestran aumento de calcio en orina de las dos poblaciones (adulta y pediátrica)

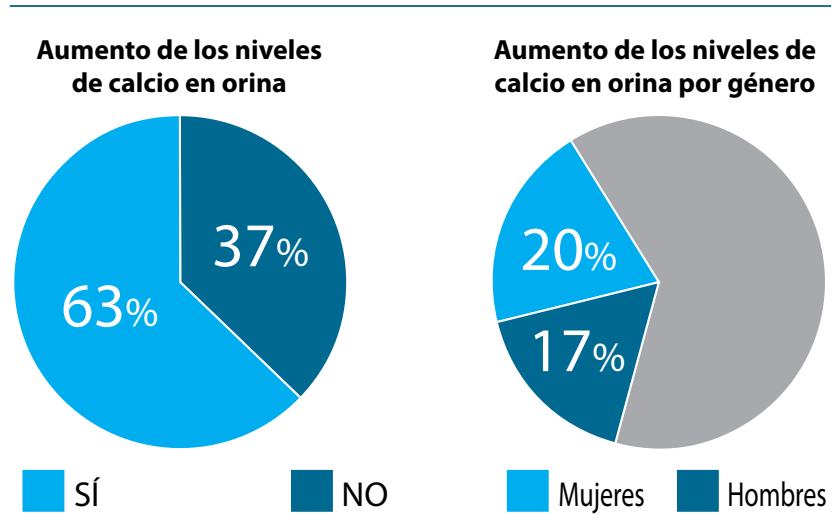

Hallazgo de los niveles de calcio elevados en muestra de orina ocasional en gran porcentaje de la población principalmente en mujeres.

tadísticamente se logró identificar que no hubo relación entre el Z-score de columna lumbar y los niveles de excreción de calcio en orina ocasional, es decir los niveles de calcio en orina en esta población no afectaron la masa ósea (figura 5). Con el fin de identificar los posibles factores relacionados con la excreción de calcio en orina, se hizo un análisis multivariado, en el que se tuvieron en cuenta variables como edad, sexo y hábitos que pueden interferir con el metabolismo del calcio, encontrándose sólo relación con el sexo, resultado que soporta que la excreción de calcio aumentada fue predominante en las mujeres que hicieron parte de este estudio (figura 6).

De acuerdo con los resultados inicialmente descritos, el $5 \%$ de la población presentó niveles de densidad mineral ósea por debajo de los parámetros normales. Se hizo un análisis para tratar de identificar si en esta población había relación 
Figura 5. Niveles de calcio en orina espontánea en Z-score ajustado a columna lumbar

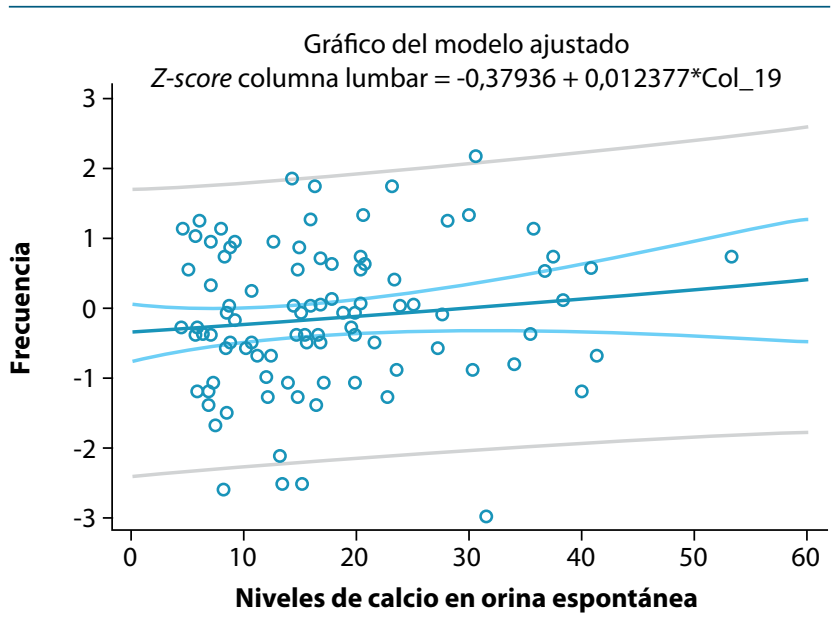

No se encontró relación directamente proporcional entre el aumento de la excreción de calcio en orina y $Z$-score de esta población.

Figura 6. Correlación entre los niveles de excreción de calcio en orina y factores relacionados con la pérdida de calcio

\begin{tabular}{l|c|c|c|c}
\hline \multicolumn{2}{l|}{} & Error & Estadístico & Estadístico \\
\cline { 5 - 6 } Parámetro & Estimación & Estándar & T & Valor-P \\
\hline Constante & $-11,4588$ & 16,3427 & $-0,701158$ & 0,4850 \\
\hline Sexo & 2,18886 & 2,35533 & 0,929323 & 0,3552 \\
\hline Consume café & 1,34651 & 2,17101 & 0,620221 & 0,5367 \\
\hline Fuma & 1,74595 & 2,91714 & 0,598514 & 0,5510 \\
\hline Fuma marihuna & 4,78368 & 5,05777 & 0,945809 & 0,3468 \\
\hline Consume alcohol & $-0,845771$ & 2,3355 & $-0,362137$ & 0,7181 \\
\hline Realiza actividad fisica & 3,59918 & 2,31478 & 1,55487 & 0,1235 \\
\hline Consume vitaminas & $-0,299213$ & 3,00197 & $-0,0996722$ & 0,9208 \\
\hline Consume complementos de calcio & 3,89024 & 6,8454 & 0,5683 & 0,5713 \\
\hline
\end{tabular}

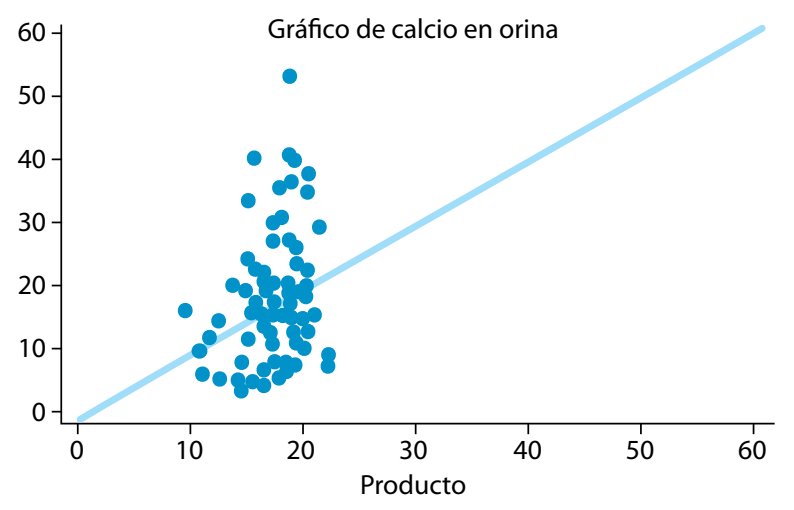

Entre ocho factores analizados que pudieran explicar la excreción aumentada de calcio en orina ocasional sólo se encontró relación con el género femenino, análisis explicado por un p-valor inferior a 0,05. del consumo de café, consumo de alcohol, realización de actividad física e índice de masa corporal con los niveles de $Z$-score de columna lumbar inferiores a -2,0. Con respecto al consumo de café se encontró que el $40 \%$ de la población con un Z-score inferior a -2,0 manifestaron consumir café regularmente (figura 7). Con respecto al consumo de alcohol se encontró que el 60\% de la población con un Z-score inferior a -2,0 manifestaron consumir licor regularmente (figura 8). Con respecto a la realización de actividad física se encontró que el $60 \%$ de la población con un Z-score inferior a -2,0 manifestaron no hacer ningún tipo de actividad física regularmente (figura 9), Con respecto al IMC se encontró que el $40 \%$ de la población con un Z-score inferior a -2,0 tenía un IMC inferior a 22, un 40\% se encontró con un IMC por encima de 25 y el $20 \%$ tenía IMC en 23 (figura 10).

Figura 7. Consumo de café en los grupos de edad

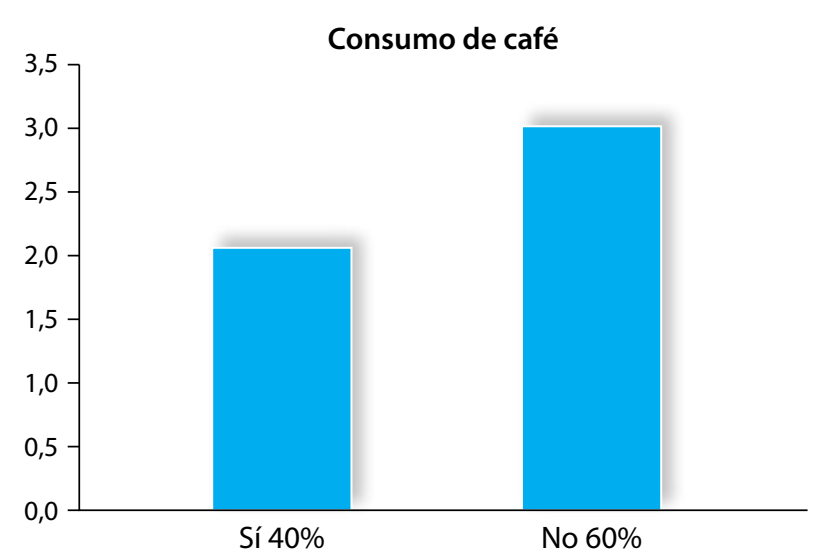

Relación de la población con niveles de Z-score de columna lumbar inferiores a $-2,0$ y el consumo de café.

Figura 8. Consumo de alcohol en los grupos de edad

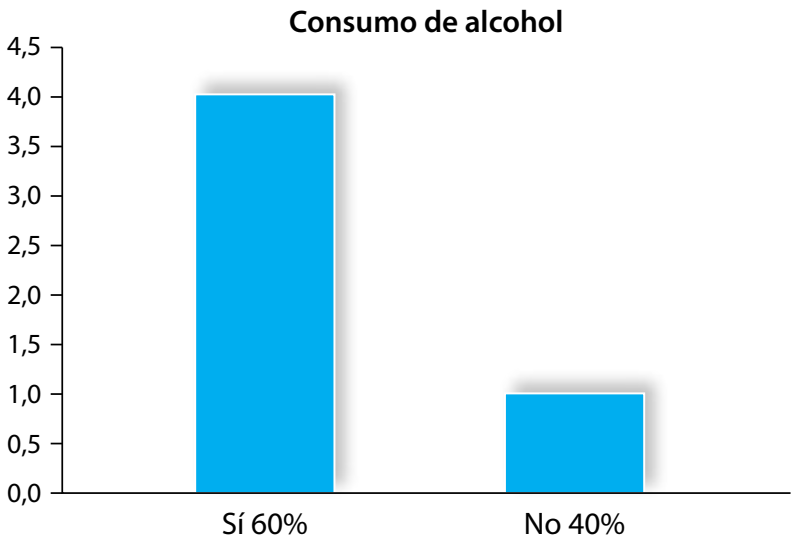

Relación de la población con niveles de Z-score de columna lumbar inferiores a $-2,0$ y el consumo de Alcohol. 
Figura 9. Realización de actividad física en los grupos de edad

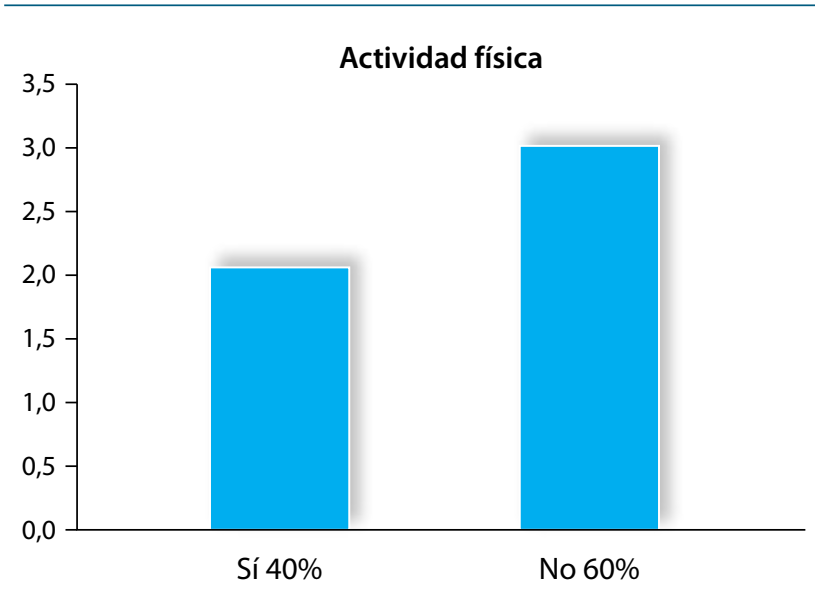

Relación de la población con niveles de Z-score de columna lumbar inferiores a $-2,0$ y la realización de actividad física.

Figura 10. Relación de la población con niveles de $Z$ score de columna lumbar inferiores a $-2,0$ y el índice de masa corporal

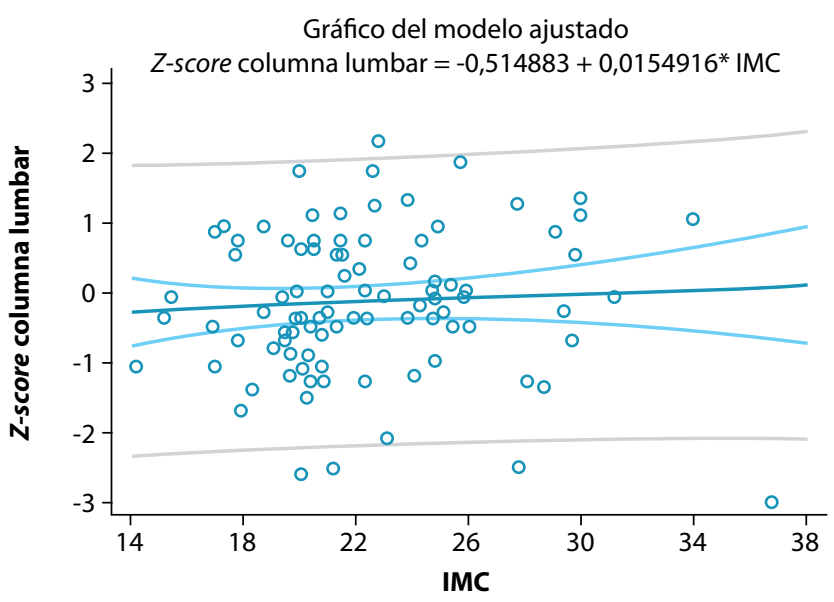

No hay evidencia en esta población de relación entre niveles de Z-score de columna lumbar inferiores a -2,0 y el índice de masa corporal.

\section{Discusión}

Para la población participante que presenta un Z-score inferior a -2,0, la densidad mineral ósea baja podría estar relacionada con la edad, el consumo de cafeína, alcohol y la realización de poca actividad física, factores que interfieren con la absorción y el metabolismo del calcio, en ausencia de otros factores de riesgo asociados. Este hallazgo es similar a un estudio realizado en la ciudad de Querétaro, México, el cual fue publicado en el 2013 y que evaluó el impacto de los factores de riesgo en osteoporosis sobre la DMO en mujeres perimenopáusicas $^{(7)}$. Como lo afirma la Bagur Calafat en el artículo "Evolución ontogénica de la masa ósea e influencia de la actividad física sobre el hueso en las diferentes etapas de la vida", la actividad física es un factor determinante sobre la salud ósea de adultos jóvenes, lo que puede contrastarse con los resultados encontrados en este estudio, donde el $60 \%$ de adultos jóvenes con DMO baja manifestaron no realizar ningún tipo de actividad física ${ }^{(1)}$, contrario a lo que reporta el American College of Rheumatology en un artículo de revisión de osteoporosis realizado en el 2012, en este estudio el consumo de cafeína alcohol y tabaco no están relacionados con los altos niveles de calcio en orina ${ }^{(3)}$. Los niveles de calcio en orina de la población sujeto no afectan la densidad mineral ósea, ni producen alteración hemodinámica, lo cual podría explicarse como un hallazgo ocasional y no como una condición patológica ${ }^{(9)}$. Hábitos prevalentes en los estudiantes universitarios como el consumo de alcohol, tabaco, cafeína y la poca actividad física son factores de riesgo para desarrollar osteoporosis en la vida adulta ${ }^{(12)}$.

Agradecimientos: A la vicerrectora de investigaciones de la corporación universitaria Alexander von Humboldt.; A la población participante y al semillero de investigación de enfermedades metabólicas del programa de Enfermería CUE AvH.

\section{Referencias}

1. Caritat Bagur C, Ejercicio físico y masa ósea (I). Evolución ontogénica de la masa ósea e influencia de la actividad física sobre el hueso en las diferentes etapas de la vida. Apunts Med Esport. 2007;42:40-6. - Vol. 42 Núm.153.

2. Heaney RP, Abrams S, Lawson-Hughes B, Looker A, Marcus R, Matkovic V, et al. Peak bone mass. Osteoporos Int. 2000;11: 985-1009.

3. Shreyasee Amin, osteoporosis, 2012 American College of Rheumatology.

4. Balance control in elderly people with osteoporosis Wei-Li Hsu, Chao-Yin Chen, Jau-Yih Tsauo, Rong-Sen Yang Journal of the Formosan Medical Association - 20 March 2014 (10.1016/j.jfma.2014.02.006).

5. Caple C, Schub T, Pravikoff D, Osteoporosis: prevención, CINAHL Nursing Guide, 2011.

6. Schurman L, bagur a, claus-hermberg h, messina o, negri a, mastaglia s, et al. guías 2012 para el diagnóstico, la prevención y el tratamiento de la osteoporosis. (Spanish). Medicina (Buenos Aires) [serial on the Internet]. (2013, Jan), [cited March 28, 2014]; 73(1): 55-74. Available from: MedicLatina.

7. Aguilera-Barreiro M, Rivera-Márquez J, Trujillo-Arriaga H, Ruiz-Acosta J, Rodríguez-García M. Impacto de los factores de riesgo en osteoporosis sobre la densi- dad mineral ósea en mujeres perimenopáusicas de la Ciudad de Querétaro, México. (Spanish). Archivos Latinoamericanos De Nutrición [serial on the Internet]. (2013, Mar), [cited March 28, 2014]; 63(1): 21-28. Available from: MedicLatina.

8. Strayer DA, Schub T, Pravikoff D, osteoporosis, CINAHL Nursing Guide, November 25, 2011.

9. Kanis JA, Melton LJ III, Christiansen C, Johnston CC, Khaltaev N. The diagnosis of osteoporosis. J Bone Miner Res 1994; 9: 1137-1141.

10. Bachrach LK, Hastie T, Wang MC, Narasimhan B, and Hispanick Black and Caucasian youth: a longitudinal study. J Clin Endocrinol Metab 84:4702-4712 2003.

11. S. Ferrari, M. L. Bianchi, J. A. Eisman, Show All (9), Osteoporosis in young adults: pathophysiology, diagnosis, and management, Osteoporosis International, 2012, Page 1.

12. M. Reyes Pérez-Fernández, Raquel Almazán Ortega, José M. Martínez Portela, M. Teresa Alves Pérez, M. Carmen Segura-Iglesias, Román Pérez-Fernández, Hábitos saludables y prevención de la osteoporosis en mujeres perimenopáusicas de un ámbito rural, Gaceta Sanitaria, Volume 28, Issue 2, March-April 2014, Pages 163 165, ISSN 0213-9111, http://dx.doi.org/10.1016/j.gaceta.2013.09.006. 